\title{
On the Homotopy Analysis Method for an Seir Tuberculosis Model
}

\author{
M.O. Ibrahim ${ }^{1}$, S.A. Egbetade ${ }^{2 * *}$ \\ ${ }^{1}$ Department of Mathematics, University of Ilorin, Ilorin, Nigeria \\ ${ }^{2}$ Department of Mathematics \& Statistics, The Polytechnic, Ibadan, Nigeria \\ *Corresponding author: egbetades@yahoo.com
}

Received August 13, 2013; Revised August 21, 2013; Accepted September 21, 2013

\begin{abstract}
In this paper, we provide a very accurate, non-perturbative, semi-analytical solution to a system of nonlinear first-order differential equations modeling the transmission of tuberculosis (TB) in a homogeneous population. Our analysis is based on Homotopy Analysis Method (HAM). Maple 15 software is used to carry out the computations. Our results show the validity and potential of HAM for computing the solution of nonlinear equations.
\end{abstract}

Keywords: uberculosis, homotopy analysis method, series solution, nonlinear equations, mathematical model

Cite This Article: M.O. IBRAHIM, and S.A. EGBETADE, "On the Homotopy Analysis Method for an Seir Tuberculosis Model." American Journal of Applied Mathematics and Statistics 1, no. 4 (2013): 71-75. doi: 10.12691/ajams-1-4-4.

\section{Introduction}

Infection with tuberculosis (TB) is caused by a bacterial known as Mycobacterium Tuberculosis [1,2,3]. Globally, $\mathrm{TB}$ is one of the greatest diseases of public concern because the pandemic is a substantial threat to socioeconomic development imposing a heavy burden on families, communities and economies [4,5,6]. In 1993, the World Health Organisation (WHO) declared TB a global emergency and about 2 billion people were estimated to be globally infected with TB that year [7]. However, with drastic global treatment measures, the incidence of TB has reduced across the globe. According to a recent global TB reports, 9.4 million people acquired the disease in 2008 resulting in 1.8 million deaths while the number of active cases has reduced to 5.7 million in 2011 with 1.4 million deaths showing that TB mortality has decreased by $22 \%$ globally since 2008 [8,9].

Mathematical models have been widely used in different forms for studying the transmission dynamics of TB epidemics [10-17]. However, the dilemma with many models in epidemiology is sometimes how to obtain analytic solutions of the nonlinear equations describing the dynamics of these diseases [18].

In 1992, a non-perturbative method known as homotopy analysis method (HAM) was proposed by Liao [19].This method was based on homotopy, an important part of topology [20]. HAM is a general analytic technique developed for the purpose of obtaining approximate analytic series solutions to different types of nonlinear equations especially those with strong nonlinearity. This method has been successfully applied to solve many types of nonlinear problems arising in the field of science, engineering and finance [21-38]. The HAM offers certain advantages over previous non-perturbative methods. Firstly, its validity does not depend upon small parameters of the considered nonlinear problem. Secondly, it provides a simple way to ensure the convergence of series solutions. Furthermore, we have great freedom to choose auxiliary linear operator so that one can approximate a nonlinear equation more efficiently by means of better base functions. Equal importantly, a few new solutions of some nonlinear problems which are neglected by all other analytic and numerical techniques are found using HAM. In addition, as proved in [25], HAM logically contains the three traditional non-perturbation methods such as Lyapunov artificial small parameter method [39], $\delta$ expansion method [40] and Adomian decomposition method [41]. The homotopy perturbation method developed in [42] is also a special case of HAM as pointed out by Sajid and Hayat [43], Liang and Jeffery [44] and other researchers.

\section{Mathematical Formulation}

In this paper, we consider the following TB epidemic model proposed by Egbetade and Ibrahim [45]

$$
\begin{gathered}
S^{\prime}=(1-\gamma) \pi+s I-\beta I S-\mu S \\
E^{\prime}=(1-\rho) \beta I S-(\mu+v) E \\
I^{\prime}=d \rho I S+D v E-\left(\mu+\mu_{T}+s\right) I \\
R^{\prime}=\varepsilon I-s I-\beta I R-\mu R
\end{gathered}
$$

where $S=$ number of susceptible who do not have the disease but could get it 
$\mathrm{E}=$ number of exposed who are infected but are yet to show any sign of symptoms

$\mathrm{I}=$ number of infectives who have the disease and can transmit it to others

$\mathrm{R}=$ number of recovered or removed who can not get the disease or transmit it.

$\gamma=$ proportion of recruitment due to immigration

$\pi=$ rate of recruitment of susceptible individuals

$\mathrm{S}=$ treatment rate of $\mathrm{TB}$

$\beta=$ transmission rate of $\mathrm{TB}$

$\mu=$ natural death rate

$\mu_{\mathrm{T}}=$ death rate of $\mathrm{TB}$

$v=$ rate of slow progression

$\rho=$ rate of fast progression

$\mathrm{D}=$ detection rate of $\mathrm{TB}$

$\varepsilon=$ rate at which susceptible individuals recover.

In section 3, we shall apply the homotopy analysis method described in the next section to solve equations (2.1) (2.4).

\section{Homotopy Analysis Method}

For the sake of completeness and readability of the present work, we give below a systematic description of the procedures of HAM.

Consider a nonlinear equation of the form

$$
N[u(t)]=0
$$

where $\mathrm{N}$ is a nonlinear operator, $\mathrm{t}$ denotes the time and $\mathrm{u}(\mathrm{t})$ is an unknown function. Let $\mathrm{u}_{0}(\mathrm{t})$ denote an initial approximation of $\mathrm{u}(\mathrm{t})$ and $\mathrm{L}$ denote an auxiliary linear operator, Liao [21] constructs the zero-order deformation equation.

$$
(1-p) L\left[\phi(t ; p)-u_{0}(t)\right]=p h H(t) N(t ; p)
$$

where $\mathrm{p} \in[0,1]$ is the embedding parameter, $\mathrm{h} \neq 0$ is a nonzero auxiliary parameter, $\mathrm{H}(\mathrm{t}) \neq 0$ is a non-zero auxiliary function.

When $\mathrm{p}=0$ and $\mathrm{p}=1$, the zero-order deformation equations becomes respectively

$$
\phi(t ; 0)=u_{0}(t)
$$

and

$$
\phi(t ; 0)=u(t)
$$

Thus, as $\mathrm{p}$ increases from 0 to 1 , the solution $\phi(\mathrm{t}$; $\mathrm{p})$ varies continuously from the initial approximation $\mathrm{u}_{0}(\mathrm{t})$ to the exact solution $u(t)$. Such a kind of continuous variation is called deformation in topology. Expanding $\phi(t ; p)$ by Taylor's series in power series of $p$, we have

$$
\phi(t ; p)=u_{0}(t)+\sum_{m=1}^{\infty} u_{m} p^{m}
$$

where

$$
u_{m}(t)=\frac{1}{m !} \frac{\partial^{m} \phi(t ; p)}{\partial p^{m}}
$$

is the deformation derivative.
If the auxiliary linear operator $\mathrm{N}$, the initial approximation $\mathrm{u}_{0}(\mathrm{t})$, the auxiliary parameter $\mathrm{h}$ and the auxiliary function $\mathrm{H}(\mathrm{t})$ are properly chosen so that

(1) the solution $\phi(t ; p)$ of the zero-order deformation equation (3.2) exists for all $p \in[0,1]$.

(2) the deformation derivative (3.6) exists for all $m=1,2, \ldots$.

(3) the series (3.5) converges at $\mathrm{p}=1$.

Then, we have the series solution

$$
\phi(t ; 1)=u_{0}(t)+\sum_{m=1}^{\infty} u_{m}(t)
$$

Define the vector

$$
\vec{u}_{m}(t)=\left\{u_{0}(t), u_{1}(t), \ldots, u_{m}(t)\right\}
$$

According to the definition (3.6), the governing equation can be derived from the zero-order deformation equation (3.2). Differentiating (3.2) $\mathrm{m}$ times with respect to the embedding parameter $p$, then setting $p=0$ and finally dividing by $m$ !, we obtain the $m$ th order deformation equation

$$
L\left[y_{m}(t)-\chi_{m} u_{m-1}(t)\right]=h H(t) Q_{m}\left(\vec{u}_{m-1}(t)\right)
$$

where

$$
Q_{m}\left(\vec{u}_{m-1}(t)\right)=\frac{1}{(m-1) !} \frac{\partial^{m-1} N[\phi(t ; p)]}{\partial p^{m-1}}
$$

and

$$
\chi_{m}= \begin{cases}0, & m \leq 1 \\ 1, & m>1\end{cases}
$$

Note that according to the definition (3.10), the right hand side of (3.9) depends only on $u_{m-1}(t)$. Thus, we easily gain the series $u_{1}(t), u_{2}(t), \ldots$ by solving the linear high-order deformation equation (3.9) using symbolic computation software such as Matlab, Maple or Mathematica.

\section{Solution of SEIR Model by HAM}

To solve the model equation (2.1) - (2.4) by HAM, we consider equation (2.1) and choose the linear operator

$$
N[S(t ; p)]=\frac{d S(t ; p)}{d t}
$$

with the property that

$$
N\left[c_{1}\right]=0
$$

where $c_{1}$ is a constant of integration. The inverse operator $N^{-1}$ is given by

$$
N^{-1}(\cdot)=\int_{0}^{t}(\cdot) d t
$$

Let the nonlinear operator be defined as 


$$
\begin{aligned}
N[S(t ; p)] & =\frac{d S(t ; p)}{d t}-(1-\gamma) \pi-s I(t ; p) \\
& +\beta I(t ; p) S(t ; p)+u S(t ; p)
\end{aligned}
$$

By constructing the zero-order deformation equation

$$
(1-p) N\left[S(t ; p)-s_{0}(t ; p)\right]=p h H(t) N[S(t ; p)](4.5)
$$

we have that for

$$
\begin{aligned}
& p=0, \text { then } S(t ; 0)=s_{0}(t) \\
& p=1, \text { then } S(t ; 1)=s(t)
\end{aligned}
$$

Then, we have the $m$ th order deformation equation

$$
N\left[S_{m}(t)-\chi_{m} S_{m-1}(A)\right]=h H(t) Q\left(\vec{S}_{m-1}(t)\right), m \geq 1(4.6)
$$

where

$$
\begin{aligned}
Q_{m}\left(\vec{S}_{m-1}(t)\right)= & \frac{d S_{m-1}(t)}{d t}-(1-\gamma) \pi-s I_{m-1}(t) \\
& +\beta I_{m-1}(t) S_{m-1}(t)+\mu S_{m-1}(t)
\end{aligned}
$$

The solution of the $m$ th order deformation equation (4.6) for $m \geq 1$ and using $h=-1$ and $H(t)=1$ is given by

$$
\begin{aligned}
S_{m}(t)= & \chi_{m} S_{m-1}(t) \\
& -\int_{0}^{t}\left(\begin{array}{l}
\frac{d}{d t} S_{m-1}(t)-(1-\gamma) \pi \\
-s I_{m-1}(t)+\beta I_{m-1}(t) S_{m-1}(t) \\
+\mu S_{m-1}(t)
\end{array}\right) d t, m \geq 1
\end{aligned}
$$

Following earlier steps, we get

$$
\begin{aligned}
E_{m}(t)= & \chi_{m} E_{m-1}(t) \\
& -\int_{0}^{t}\left(\begin{array}{l}
\frac{d}{d t} E_{m-1}(t) \\
-(1-\rho) \beta I_{m-1}(t) S_{m-1}(t) \\
+(\mu+v) E_{m-1}(t)
\end{array}\right) d t, m \geq 1
\end{aligned}
$$

$$
\begin{aligned}
& I_{m}(t)=\chi_{m} I_{m-1}(t) \\
&-\int_{0}^{t}\left(\begin{array}{l}
\frac{d}{d t} I_{m-1}(t)-d \rho \beta I_{m-1}(t) S_{m-1}(t) \\
-d v E_{m-1}(T) \\
-\left(\mu+\mu_{T}+\varepsilon\right) I_{m-1}(t)+s I_{m-1}(t)
\end{array}\right) d t, m \geq 1
\end{aligned}
$$

$$
\begin{aligned}
R_{m}(t)=\chi_{m} R_{m-1}(t) \\
-\int_{0}^{t}\left(\begin{array}{l}
\frac{d}{d t} R_{m-1}(t)-\varepsilon I_{m-1}(t) \\
+s I_{m-1}(t) \\
+s I_{m-1}(t) R_{m-1}(t)+\mu R_{m-1}(t)
\end{array}\right) d t, m \geq 1
\end{aligned}
$$

\section{Numerical Results and Discussion}

For numerical results, the following values for parameters are considered.
Table 1. Parameter values for the series solutions

\begin{tabular}{|c|c|}
\hline Parameter & Assigned values \\
\hline S & 20 \\
\hline E & 10 \\
\hline I & 15 \\
\hline R & 5 \\
\hline$\beta$ & 0.02 \\
\hline$\gamma$ & 0.08 \\
\hline $\mathrm{s}$ & 0.2 \\
\hline$\mu$ & 0.1 \\
\hline$\mu_{\mathrm{T}}$ & 0.03 \\
\hline $\mathrm{U}$ & 0.04 \\
\hline$\varepsilon$ & 0.3 \\
\hline $\mathrm{d}$ & 0.4 \\
\hline$\pi$ & 0.3 \\
\hline$\rho$ & 0.05 \\
\hline
\end{tabular}

For high accuracy of results, we use Maple 15 computation software [46].For the graphs, dot lines: Susceptibles; dash lines: Exposed; dashdot lines: Infectives; longdash lines: Recovered. The $5^{\text {th }}, 6^{\text {th }}, 7^{\text {th }}$ and $8^{\text {th }}$ terms approximations for $\mathrm{S}(\mathrm{t}), \mathrm{E}(\mathrm{t}), \mathrm{I}(\mathrm{t})$ and $\mathrm{R}(\mathrm{t})$ are calculated and presented below.

\section{$5^{\text {th }}$ terms approximations}

$$
\begin{aligned}
S_{5}(t)= & 20-4.724 t+0.5782 t^{2}+0.00844498667 t^{3} \\
& -0.00251226171 t^{4}-0.00034215451 t^{5} \\
E_{5}(t)= & 10+4.46 t-0.266284 t^{2}+0.0116749093 t^{3} \\
& +0.00338895936 t^{4}-0.0006101162407 t^{5} \\
I_{5}(t)= & 15+3.66 t+0.4487436 t^{2}+0.03337370672 t^{3} \\
+ & 0.00194694054 t^{4}+0.000106838353 t^{5} \\
R_{5}(t)= & 5-0.5 t+0.1 t^{2}-0.00111333333 t^{3} \\
& -0.000599807669 t^{4}+0.0000090582606 t^{5}
\end{aligned}
$$

\section{$6^{\text {th }}$ terms approximations}

$$
\begin{aligned}
S_{6}(t) & =20-4.724 t+0.5782 t^{2} \\
& +0.00844498667 t^{3}-0.00251226171 t^{4} \\
& -0.00034215451 t^{5}-0.00005700487683 t^{6} \\
E_{6}(t)= & 10+4.46 t-0.266284 t^{2} \\
+ & 0.0116749093 t^{3}+0.00338895936 t^{4} \\
- & 0.0006201162407 t^{5}+0.00001953571468 t^{6} \\
I_{6}(t)= & 15+3.66 t+0.4487436 t^{2} \\
+ & 0.03337370672 t^{3}+0.00194694054 t^{4} \\
+ & 0.000106838353 t^{5}+0.00000197134589 t^{6} \\
R_{6}(t)= & 5-0.5 t+0.1 t^{2} \\
- & 0.00111333333 t^{3}-0.000599807669 t^{4} \\
+ & 0.0000090582606 t^{5}+0.00000235519466 t^{6}
\end{aligned}
$$

\section{$7^{\text {th }}$ terms approximations}




$$
\begin{aligned}
S_{7}(t) & =20-4.724 t+0.5782 t^{2}+0.00844498667 t^{3} \\
& -0.00251226171 t^{4}-0.00034215451 t^{5} \\
& -0.00005700487683 t^{6},+0.0000106219886 t^{7} \\
E_{7}(t)= & 10+4.46 t-0.266284 t^{2}+0.0116749093 t^{3} \\
+ & 0.00338895936 t^{4}-0.0006201162407 t^{5} \\
+ & 0.00001953571468 t^{6}-0.000006581417588 t^{7} \\
I_{7}(t) & =15+3.66 t+0.4487436 t^{2}+0.03337370672 t^{3} \\
+ & 0.00194694054 t^{4}+0.000106838353 t^{5} \\
+ & 0.00000197134589 t^{6}-0.00009629017459 t^{7} \\
R_{7}(t) & =5-0.5 t+0.1 t^{2}-0.00111333333 t^{3} \\
& -0.000599807669 t^{4}+0.0000090582606 t^{5} \\
& +0.00000235519466 t^{6}+0.00005687282 t^{7}
\end{aligned}
$$

\section{$8^{\text {th }}$ terms approximations}

$$
\begin{aligned}
S_{8}(t) & =20-4.724 t+0.5782 t^{2} \\
& +0.00844498667 t^{3}-0.00251226171 t^{4} \\
& -0.00034215451 t^{5}-0.00005700487683 t^{6} \\
& +0.0000106219886 t^{7}+0.000049876956 t^{8} \\
E_{8}(t)= & 10+4.46 t-0.266284 t^{2} \\
+ & 0.0116749093 t^{3}+0.00338895936 t^{4} \\
+ & 0.000106838353 t^{5}+0.00000197134589 t^{6} \\
& -0.00009629017459 t^{7}+0.00000286668947 t^{8} \\
I_{8}(t)= & 15+3.66 t+0.4487436 t^{2} \\
+ & 0.03337370672 t^{3}+0.00194694054 t^{4} \\
+ & 0.000106838353 t^{5}+0.00000197134589 t^{6} \\
- & 0.00009629017459 t^{7}+0.000002866668947 t^{8}
\end{aligned}
$$

$$
\begin{aligned}
R_{8}(t) & =5-0.5 t+0.1 t^{2} \\
& -0.00111333333 t^{3}-0.000599807669 t^{4} \\
& +0.0000090582606 t^{5}+0.00000235519466 t^{6} \\
& +0.00005687282 t^{7}+0.00000127768296 t^{8}
\end{aligned}
$$

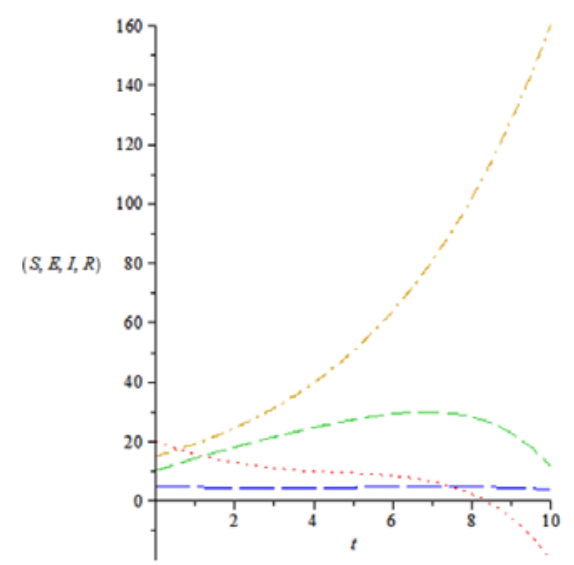

Figure 1. Plots of $5^{\text {th }}$ terms approximations for $\mathrm{S}(\mathrm{t}), \mathrm{I}(\mathrm{t})$ and $\mathrm{R}(\mathrm{t})$ against time $(\mathrm{t})$

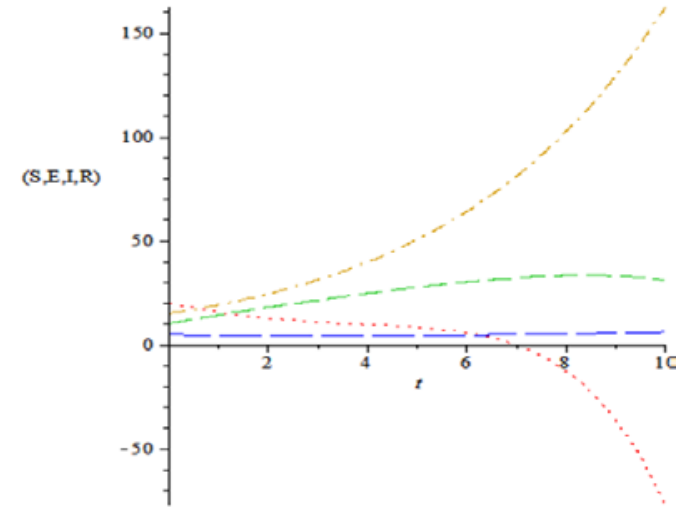

Figure 2. Plots of $6^{\text {th }}$ terms approximations for $\mathrm{S}(\mathrm{t})$, I $\mathrm{I}(\mathrm{t})$ and $\mathrm{R}(\mathrm{t})$ against time $(\mathrm{t})$

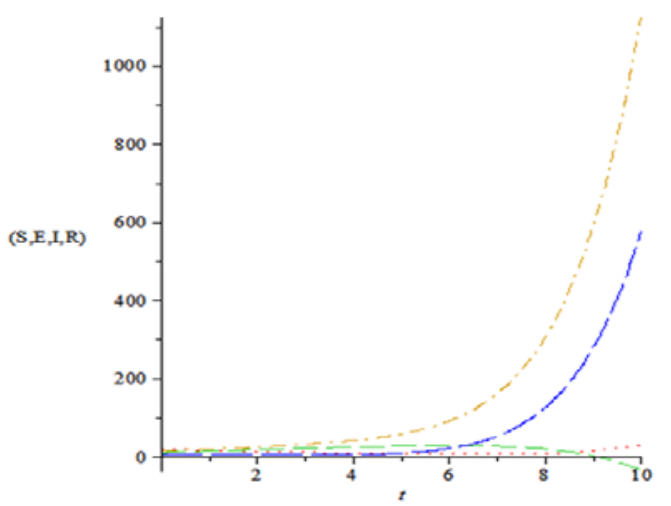

Figure 3. Plots of $7^{\text {th }}$ terms approximations for $\mathrm{S}(\mathrm{t}), \mathrm{E}(\mathrm{t}), \mathrm{I}(\mathrm{t})$ and $\mathrm{R}(\mathrm{t})$ against time(t)

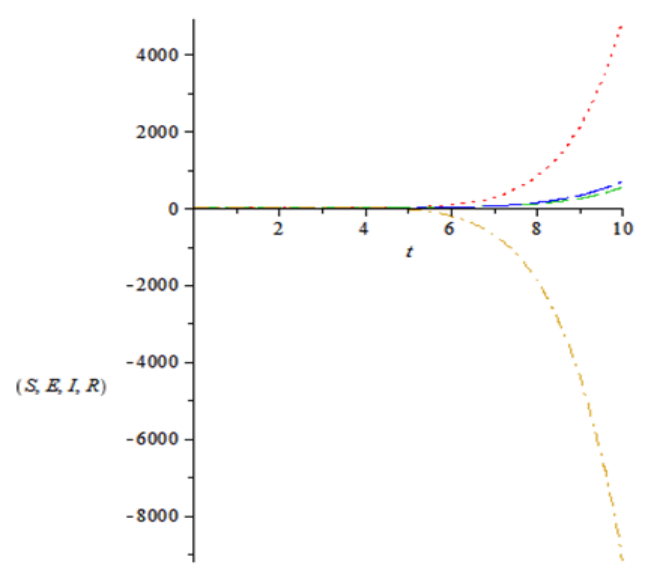

Figure 4. Plots of $8^{\text {th }}$ terms approximations for $\mathrm{S}(\mathrm{t}), \mathrm{E}(\mathrm{t}), \mathrm{I}(\mathrm{t})$ and $\mathrm{R}(\mathrm{t})$ against time(t)

From the various order of approximations, the HAM yields convergent series solutions that are reasonable and easy to express. The plots show that while the number of susceptible (S) decreases the population who are infectives (I) increases in the period of the epidemic. Meanwhile, the number of exposed (E) increases while the number of recovered (R) decreases. However, from figure 4 , as the infection dies out (i.e. as $I \rightarrow 0$, the number of susceptible, exposed and recovered increases. In particular, as $\mathrm{I} \rightarrow 0, \mathrm{~S}$ approaches some positive value $\mathrm{S}=6.0513$ which is the eventual population who were never infective.

\section{Conclusion}

In this paper, the HAM has been successfully applied to approximately solve a system of nonlinear equations in 
tuberculosis dynamics. The results show the potential and efficiency of HAM in solving nonlinear problems. We thus conclude that, combined with high performance computer and symbolic computation software such as Maple and so on, the homotopy analysis method might become a new powerful analytic tool to get satisfactory approximations for nonlinear problems in science and engineering.

\section{References}

[1] Blower, S.M., McLean, A.R., Porco, T.C., Small, P.M., Hopewell, P.C., Sanchez, M.A. and Moss, A.R., " The intrinsic transmission dynamics of tuberculosis epidemics,” Nat. Med., 1(8). 815-821. 1995.

[2] Song, B., Castillo-Chavez, C. and Aparicio, J-P., "Tuberculosis with fast and slow dynamics: the role of close and casual contact," Math. Biosci., 180. 187-205. 2002.

[3] Egbetade, S.A. and Ibrahim, M.O., "Global stability results for a tuberculosis epidemic model,” Res. J. Maths \& Stat., 4(1). 14-20. 2012.

[4] Colijn, C., Cohen, T. and Murray, M. (2006) Mathematical models of tuberculosis: accomplishments and future challenges. Proc. Natl. Acad. USA 103(18), 1-28.

[5] World Health Organisation . Tuberculosis factsheet. 2010. http://www.who.int/medicenter/factsheet/html.

[6] Ibrahim, M.O., Ejieji, C.N. and Egbetade, S.A., "A mathematical model for the epidemiology of tuberculosis with estimate of the basic reproduction number," IOSR J. of Maths., 5(5). 46-52. 2013.

[7] World Health Organisation. Tuberculosis control. 2009. http://who/globalreport2009/pdf.

[8] World Health Organisation. WHO global TB report.2012. www.int/tb/publications/factsheet.

[9] Egbetade, S.A., Ibrahim, M.O. and Ejieji, C.N., "On existence of a vaccination model of tuberculosis disease pandemic," Int. J. Engrg. and Sc., 2(7). 41-44. 2013.

[10] Waaler, H., Geser, A. and Anderson, S., "The use of mathematical models in the study of the epidemiology of tuberculosis," Am. J. Public Health, 52. 1002-1013.1062.

[11] Castillo-Chavez, C. and Feng, Z., "To treat or not to treat: the case of tuberculosis,” J. Math. Biol., 35(6). 629-656. 1997.

[12] Aparicio, J.P., Capurro, A.F. and Castillo-Chavez, C., "Transmission and dynamics of tuberculosis on generalized households,” J. Theor. Biol., 206. 327-341. 2000.

[13] Dye, C., "Global epidemiology of tuberculosis," Lancet, 367(9514). 938-940.2006.

[14] Cohen, T., Colijn, C., Finklea, B. and Murray, M. "Exogeneous re-infection and the dynamics of tuberculosis epidemics: local effects in a network model of transmission," J.R. Soc. Interface, 4(14). 523-531.2007.

[15] Roeger, L.W., Feng, Z. and Castillo-Chavez, C. "Modeling TB and HIV co-infections," Mathematical Biosciences and Engineering, 6(4). 815-837. 2009.

[16] White, P.J. and Garnett, G.P., "Mathematical modeling of the epidemiology of tuberculosis," In: Michael, E., Speer, R.C. eds. Advances in Experimental Medicines and Biology Vol. 673, Modeling Parasite Transformation and Control. NY:Springer+Business Media, LLC Landes Biosciences, 127-140.

[17] Oxlade, O., Sterling, T.R. and Schwartzman, K., "Developing a tuberculosis transmission model that accounts for changes in population health,” Medical Decision Making, 31. 53-68. 2011.

[18] Brauer, F., "Basic ideas of mathematical epidemiology. Mathematical approaches for energy and re-emerging infectious diseases: Models, Methods and Theory,” (eds. Castillo-Chavez, C., Blower, S., van der Driessche, P., Kirschner, D. and Yakubu, A.A.). Berlin, Springer-Verlag.

[19] Liao, S.J., The proposed homotopy analysis method for the solutions of nonlinear problems. Ph.D. Thesis. Shanghai Jiao Tong University, Shanghai, China.1992.

[20] Alexander, J.L. and York, J.A., "The homotopy continuation method: numerically implementable topological procedures," Trans Am. Math. Soc., 242. 271-284. 1978.
[21] Liao, S.J., "A kind of approximate solution technique which does not depend upon small parameters (II): an application in fluid mechanics,” Int. J. Nonlinear Mech., 32(5). 815-822. 1997.

[22] Liao, S.J. and Chwang, A.T., "Application of homotopy analysis method in nonlinear oscillations,” Trans. ASME J. Appl. Mech., 65. 914-922. 1998.

[23] Liao, S.J., "An explicit totally analytic approximate solution for Blasius viscous flow problems,” Int. J. Nonlinear Mechanics, 34. 759-778. 1999.

[24] Liao, S.J., "Analytic approximation of the drag coefficient for the viscous flow past a sphere," Int. J. Nonlinear Mech., 37, 118.2002.

[25] Liao, S.J.2003 Beyond Perturbation: Introduction to the homotopy analysis method. Chapman and Hall, CRC Press, Boca Raton.

[26] Liao, S.J., "On the analytic solution of magnetohydrodynamic flow of non-Newtonian fluids over a stretching sheets," J. Fluid Mech., 488, 189-212. 2003.

[27] Liao, S.J. and Magyari, E., "Exponentially decaying boundary layers and limiting cases of families of algebraically decaying ones," ZAMP, 57(5). 777-792. 2006

[28] Hayat, T., Khan, M. and Asghar, S., "Homotopy analysis of MHD flows of an Oldroyd 8-constant fluid," Acta. Mech., 168. 213-232. 2004.

[29] Zhu, S.P., "An exact explicit solution for the evaluation of American put options," Quantitative Finance, 6. 229-242. 2006.

[30] Zhu, S.P., "A closed form analytical solution for the evaluation of convertible bonds with constant dividend yield," Anzian J., 47, 477-494. 2006.

[31] Song, H. and Tao, L., "Homotopy analysis of 1D unsteady nonlinear ground water flow through porous media," J. Coastal Res., 50. 292-295. 2007.

[32] Abbasbandy, S., "Application of homotopy analysis method to solve a generalized Hirota-Satsuma coupled KdV equation," Phys. Lett. A, 361. 478-483. 2007.

[33] Awawdeh, F., Adawi, A. and Mustafa, Z., "Solutions of the SIR models of epidemics using HAM," Chaos, Solitons and Fractals, 42. 3047-3052. 2009.

[34] Matinfar, M. and Saeidy, M., "Application of homotopy analysis method to fourth order parabolic partial differential equations," Applications and Applied Mathematics, 5(9).70-80. 2010.

[35] Li, Y., Nohara, B.T. and Liao, S.J., "Series solutions of coupled Van der Pol equation by means of homotopy analysis method," Journal of Mathematical Physics, 51. 063517. 2010.

[36] Hassan, H.N. and El-Tawil, M.A., "A new technique of using homotopy analysis method for solving high-order nonlinear differential equations," Mathematical methods in Applied Science, 34. 728-742. 2011.

[37] Arafa, A.A.M., Rida, S.Z. and Khalil, M., "Solutions of fractional order model of childhood diseases with constant variation strategy,” Math. Sc. Lett., 1(1). 17-23. 2012.

[38] Vahdati, S., Tavassoli, K.M. and Ghasemi, M., "Application of homotopy analysis method to SIR epidemic model," Research Journal of Recent Sciences, 2(1). 91-96. 2013.

[39] Lyapunov, A.M., "General problems on stability of motion," Taylor and Francis, London.

[40] Awrejcewicz, J., Andrianov, I.V. and Manevitch, L.I., "Asymptotic approaches in nonlinear dynamics," Springer-Verlag, Berlin.

[41] Adomian, G., "A review of the decomposition method and some recent results for nonlinear equations," Comp. Math. Appl., 21. 101-127. 1991.

[42] He, J.H., "Homotopy perturbation techniques," Comput. Methods Appl. Mech. Engrg., 178. 257-262. 1999.

[43] Sajid, M. and Hayat, T., "Comparison of HAM and HPM methods for nonlinear heat conduction and convection equations," Nonlinear Anal.: Real World Appl., 9. 2296-2301. 2008.

[44] Liang, S.X. and Jeffery, D.J., "Comparison of homotopy analysis method and homotopy perturbation method through an evaluation equation," Comm. Nonlinear Sci. Numer. Simul., 14. 4057-4064. 2009.

[45] Egbetade, S.A. and Ibrahim, M.O., "Stability analysis of equilibrium states of an SEIR tuberculosis model,” Journal of the Nigerian Association of Mathematical Physics, 20. 119-124. 2012.

[46] Maplesoft 15, Waterloo Maple Inc. Ontario, Canada. 2011. 